ThE AstrophysiCAL Journal, 424:L13-L16, 1994 March 20

(C) 1994. The American Astronomical Society. All rights reserved. Printed in U.S.A.

\title{
OPTICAL SIGNATURES OF HIGH-REDSHIFT GALAXY CLUSTERS
}

\author{
August E. Evrard \\ Physics Department, University of Michigan, Ann Arbor, MI 48109 \\ AND \\ STÉPHANE CHARLOT ${ }^{1}$ \\ Astronomy Department, University of California, Berkeley, CA 94720 \\ Received 1993 July 8; accepted 1994 January 7
}

\begin{abstract}
We combine an $N$-body and gasdynamic simulation of structure formation with an updated population synthesis code to explore the expected optical characteristics of a high-redshift cluster of galaxies. We examine a poor $(2 \mathrm{keV})$ cluster formed in a biased, cold dark matter cosmology and employ simple, but plausible, threshold criteria to convert gas into stars.

At $z=2$, the forming cluster appears as a linear chain of very blue $(g-r \simeq 0)$ galaxies, with 15 objects brighter than $r=25$ within a 1 square arcmin field of view. After 2 Gyr of evolution, the cluster viewed at $z=1$ displays both freshly infalling blue galaxies and red galaxies robbed of recent accretion by interaction with the hot intracluster medium. The range in $G-R$ colors is $\sim 3$ mag at $z=1$, with the reddest objects lying at sites of highest galaxy density. We suggest that red, high-redshift galaxies lie in the cores of forming clusters and that their existence indicates the presence of a hot intracluster medium at redshifts $z \simeq 2$.

The simulated cluster viewed at $z=2$ has several characteristics similar to the collection of faint, blue objects identified by Dressler et al. in a deep Hubble Space Telescope observation. The similarities provide some support for the interpretation of this collection as a high-redshift cluster of galaxies.

Subject headings: cosmology: theory - galaxies: clusters of - galaxies: evolution — galaxies: formation
\end{abstract}

\section{INTRODUCTION}

The character and abundance of clusters of galaxies at high redshift can, in principle, provide strict constraints on cosmological models of structure formation (e.g., Kaiser 1986). The initerpretation of cluster observations at optical or X-ray wavelengths, however, requires detailed theoretical modeling. The models must take into account changes in all relevant components, such as dark matter, hot intracluster gas, and cold gas and stars within galaxies. In particular, the description of star formation and of the evolution of the stellar populations in galaxies is essential for predicting the optical appearance of distant clusters. There is empirical evidence for changes in the stellar populations of cluster galaxies as one looks to more distant clusters. As first noted by Butcher \& Oemler (1978), the optical color-magnitude diagrams of distant clusters generally show a sequence of red galaxies, thought to be the analogs of present-day E/S0 galaxies, and significant numbers of blue galaxies with no known counterparts in low-redshift clusters such as the Coma Cluster. The fraction of these blue galaxies appears to increase with redshift. In very distant $(0.7 \lesssim z \lesssim 0.9)$ clusters, the red-sequence galaxies may be systematically bluer, consistent with viewing aging stellar populations formed prior to $z \simeq 2$ (Dressler \& Gunn 1990; Aragón-Salamanca et al. 1993). So far, only a handful of clusters have been tentatively identified at redshifts $z \gtrsim 1$ (Hintzen, Romanishin, \& Valdes 1991; Dressler et al. 1993; Dickinson 1993).

In this Letter, we present theoretical predictions of optical images of a high-redshift cluster using an approach that employs two existing tools. We combine a high-resolution twofluid simulation of a small cluster of galaxies in a cold dark

\footnotetext{
${ }^{1}$ Also affiliated with the Center for Particle Astrophysics, University of California at Berkeley and the Institute of Geophysics and Planetary Physics, Lawrence Livermore National Laboratory.
}

matter (CDM) universe (Evrard, Summers, \& Davis 1994, hereafter ESD) with an updated population synthesis code (Bruzual \& Charlot 1993, hereafter BC). The combination allows self-consistent modeling of the star-formation rate and galactic/cluster dynamics. The basic physical processes included are (1) gravitational dynamics of a coupled system of dark matter and baryons, (2) baryon dissipation via radiative cooling with subsequent conversion of gas into stars, and (3) evolution of the stars $(\S 2)$.

There are, unavoidably, a large number of tunable parameters in such a study. Along with the usual cosmological parameters, we must also introduce star-formation criteria and specify stellar population details, such as the initial mass function and metallicity. Although desirable in principle, a thorough parameter space study is currently impractical. This Letter endeavors to demonstrate the viability of our approach using parameters set to what we consider "most likely" values. Previous similar work includes the study by Baron \& White (1987) on the $L y \alpha$ properties of protogalactic clouds and the work on disk galaxy formation by Katz (1992). Both studies analyzed a small number of individual galaxies. Our focus here is a small group comprised of several tens of galaxies grown in a "natural" cosmological setting containing several hundred. We present expected magnitudes and colors of cluster galaxies at $z=2$ and 1 and examine differences between the cluster and field galaxy stellar populations $(\S 3)$. We then compare our expectations with the candidate cluster of "nascent," blue galaxies found serendipitously by Dressler et al. (1993) around a QSO at $z=2.055$ in a deep Wide-Field Camera exposure with the Hubble Space Telescope (HST) (§ 4).

\section{OPTICAL IMAGES OF HIGH-REDSHIFT CLUSTERS}

To model the galactic dynamics, we employ the two-fluid simulation of structure formation described in ESD. The simu- 
lation models a comoving $16 \mathrm{Mpc}$ cube with 524,288 particles, half representing dark matter and half representing baryons. A CDM cosmology is assumed with $\Omega=1, H_{0}=50 \mathrm{~km} \mathrm{~s}^{-1}$ $\mathrm{Mpc}^{-1}$ and baryon fraction $\Omega_{b}=0.1$. The baryons are modeled as an ideal gas with thermal energy changes due to $P d V$ work, shock heating and radiative cooling based on collisional ionization equilibrium rates. The mass per baryon particle is $1.08 \times 10^{8} M_{\odot}$, implying an $L_{*}$ galaxy is resolved by roughly 1000 particles. The minimum effective spatial resolution of the experiment is $\sim 13 \mathrm{kpc}$. The initial pseudorandom density field was constrained using Bertschinger's (1987) technique to generate a $3 \sigma$ density enhancement on a mass scale $4 \times 10^{13} M_{\odot}$ near the center of the volume. The system evolution was halted at $z=1$ partly because of CPU limitations and partly because the largest waves in the simulated volume were approaching nonlinearity (see ESD).

ESD examined characteristics of a population of galaxy tracers termed globs ("galaxy-like objects"), defined as baryonic clumps above a fixed physical density of $\sim 0.5 \mathrm{~cm}^{-3}$. Dissipation via radiative cooling leads to a distinct two-phase medium in collapsed regions, with lumps of cold, dense gas embedded in a halo of hot, tenuous material. Many of the larger globs possess extended, centrifugally supported disks of cold gas. A total of 203 globs were found above a 32 particle $\left(3 \times 10^{9} M_{\odot}\right)$ mass limit at $z=1$. Star formation was not explicitly included in the simulation. Here, we model star formation a posteriori by following trajectories of particles in the density-temperature $(n-T)$ plane and using combined thresholds in $n$ and $T$ to determine the star-formation epoch of a given particle. Analysis of the phase filling factors done in ESD reveals a distinct spike of material at densities $n>0.5 \mathrm{~cm}^{-3}$ and temperatures $T<3 \times 10^{4} \mathrm{~K}$ containing $\sim 20 \%$ of the baryons at $z=1$. We adopt these thresholds as our starformation criteria with the aim of capturing the material that winds up in the glob population. Accordingly, a total of 47,627 "star" particles (18\% of the baryons) form by $z=1$, with $30,030(11 \%)$ in place by $z=2$.

Our results are only weakly sensitive to the adopted density and temperature thresholds for star formation; the cooling instability is such that material in halos with short cooling times moves to occupy this corner of phase space in roughly one dynamical time. The result is a "desert" in phase space separating the cold, collapsed component from the hot, collapsed phase with long cooling times (see Fig. 5 of ESD). Note, however, that the finite resolution of the simulation implies that baryonic lumps less massive than $\sim 3 \times 10^{9} M_{\odot}$ do not form stars. The star formation efficiency in low-mass galaxies is expected to be small due to the combined effects of supernova feedback (e.g., Dekel \& Silk 1986) and photoionization heating (Cen \& Ostriker 1992; Efstathiou 1992).

We compute the spectral evolution of star particles using the $\mathrm{BC}$ models of stellar population synthesis. These include recent advances in the theories of stellar interiors and atmospheres. The models have solar metallicity and account for all phases of stellar evolution from the zero-age main sequence to supernova explosion (for progenitors more massive than $8 M_{\odot}$ ) or the end of the white-dwarf cooling sequence (for less massive progenitors). The main adjustable parameters in the population synthesis models are the star-formation rate and the initial mass function. Here, we assume that a particle in the simulation is turned into stars instantaneously when it meets the star-formation criteria given above, although similar results would be obtained by adopting any finite timescale of star formation smaller than the output time intervals of the simulation (i.e., $\lesssim 4 \times 10^{7} \mathrm{yr}$ ). We adopt the standard Salpeter initial mass function, defined such that the number of stars born with masses between $m$ and $m+d m$ is $\propto m^{-2.35}$, with lower and upper cutoffs, respectively, of $0.1 M_{\odot}$ and $100 M_{\odot}$. This defines the spectral evolution of a star particle at ultraviolet to near-infrared wavelengths (see Fig. $4 a$ of BC). We then construct broad-band photometric images of the simulated cluster at a given redshift by summing the $K$-corrected fluxes from star particles along lines of sight in the simulated volume defined by the pixels in a fictitious observing window. We do not include extinction of the stellar radiation by dust in our calculations. Increasing or decreasing the metallicity of the models by a factor of 2 would, respectively, redden or blue the predicted $g-r$ colors of objects at $z \gtrsim 1$ by only about 0.1 mag (Charlot \& Silk 1993).

\section{RESULTS}

We present in Figure 1 (Plate L2) images corresponding to $64 \times 64 \operatorname{arcsec}^{2}$ fields centered on the simulated cluster at $z=2$ and $z=1$ in the Gunn $g, r$, and $i$ filters. For our assumed cosmology with $q_{0}=0.5$ and $h=0.5,1^{\prime \prime}$ corresponds to a physical scale of $\sim 8 \mathrm{kpc}$ at both $z=1$ and 2 . The $13 \mathrm{kpc}$ effective resolution length of the simulation translates to an angular scale of 1".6. Sizes of the simulated globs are at best upper limits, since higher resolution experiments may allow higher concentration of the cold baryon component. Features such as optical extents and surface brightnesses may therefore be considered as upper and lower limits, respectively. Integral quantities such as total magnitudes and colors are more robust. At $z=2$, the dynamically young cluster appears as a linear chain of 15 objects with $r<25$. The linear configuration arises from infall along filaments oriented in a plane parallel with the viewing direction. Under the standard spherical model assumption that the virialized portion of the cluster be defined by the radius at which the interior density contrast is $\sim 170$, the cluster at $z=2$ has a physical virial radius $R=270 \mathrm{kpc}$ and total mass $M=2.5 \times 10^{13} M_{\odot}$, and the glob velocity dispersion is $\sigma=380 \mathrm{~km} \mathrm{~s}^{-1}$. The field of view roughly encompasses the virialized portion of the cluster. In the discussion below, we take cluster members to be objects which lie within the three-dimensional virial region defined by the 170 density contrast sphere. The entire length of the simulation is projected in Figure 1; thus, some objects are foreground and background contaminants. No attempt has been made to add objects outside of the simulated volume.

By $z=1$, the simulated cluster has grown to size $R=530$ kpc and total mass $M=6.1 \times 10^{13} M_{\odot}$, with roughly fixed velocity dispersion $\sigma=360 \mathrm{~km} \mathrm{~s}^{-1}$. The region shown in Figure 1 is the inner half of the virial region, which contains 19 objects brighter than $r=25$. This is similar to the number of galaxies visible at the same limiting magnitude at $z=1$. However, the morphology of the cluster is no longer linear, due to relaxation and mixing of glob orbits. Another evident difference between the $z=2$ and $z=1$ images is the range of colors. Figure 2 shows color-magnitude diagrams for the cluster fields, using total magnitudes based on the stars within $30 \mathrm{kpc}$ of each glob center. Also shown in the figure are colors of objects in random fields away from the cluster center. For reference, the color range spanned by present-day, $K$-corrected irregular and elliptical galaxies would be $-0.1 \lesssim g-r \lesssim 0.5$ at $z=2$ and $-0.2 \lesssim g-r \lesssim 2.8$ at $z=1$ (for an elliptical galaxy with a mild UV upturn; see Fig. 5 of BC). At $z=2$, the globs in the 

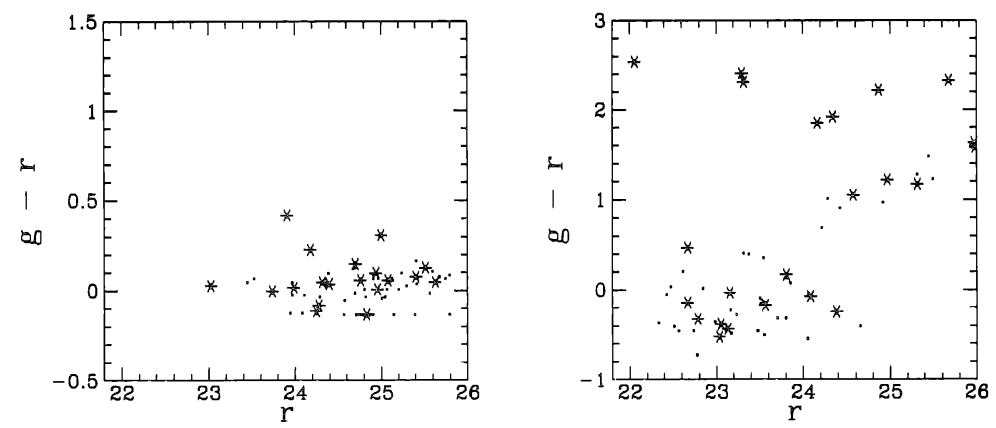

FIG. 2.-Color-magnitude diagrams of the simulated galaxies at (left) $z=2$ and (right) $z=1$ for objects in the cluster field shown in Fig. 1 (asterisks) and objects in random, noncluster fields (dots)

simulated cluster have a median $g-r$ color near 0.0 , with the reddest $g-r \simeq 0.4$, close to the value expected for galaxies dominated by evolved stellar populations. Field galaxies have a median color about $0.1 \mathrm{mag}$ bluer and are all bluer than $g-r=0.2$. At $z=1$, the median color for globs with $r \leq 25$ mag in the cluster is still fairly blue at $g-r=0.15$, but a red sequence of objects has appeared with colors typical of passively evolving galaxies. The random fields, on the other hand, contain no bright red objects. We note that if our simulated globs are allowed to passively evolve beyond $z=1$, their $g-r$ colors are slightly ( $\lesssim 0.1 \mathrm{mag}$ ) redder than the reddest objects observed by Dressler \& Gunn (1992) in clusters at redshifts $0.3 \lesssim z \lesssim 0.6$.

The red globs in the cluster are those which have had gas accretion shut off by interaction with the hot intracluster medium. As the cluster develops, the complex flow field of the infalling gas leads to shocks that heat ambient gas surrounding globs to the cluster virial temperature $T \simeq 2 \mathrm{keV}$. The ram pressure of the intracluster medium can remove the gas reservoir surrounding an infalling galaxy, preventing further accretion and star formation. This is the classic picture for the formation of S0 galaxies (Larson, Tinsley, \& Caldwell 1980). The expectation, then, is that the reddest galaxies should be found in regions of high local density as measured, for example, by the local galaxy number density.

Figure 3 shows that, in fact, the reddest $g-r$ colors at $z=1$ are related to local density contrast $\Delta$ in the expected manner, but color as a function of density is not single-valued. Here, $\Delta$ is the three-dimensional number density contrast in globs determined by the distance to the sixth nearest glob (see ESD). The asterisks at $\Delta=10$ in the cluster field in Figure 3 are along

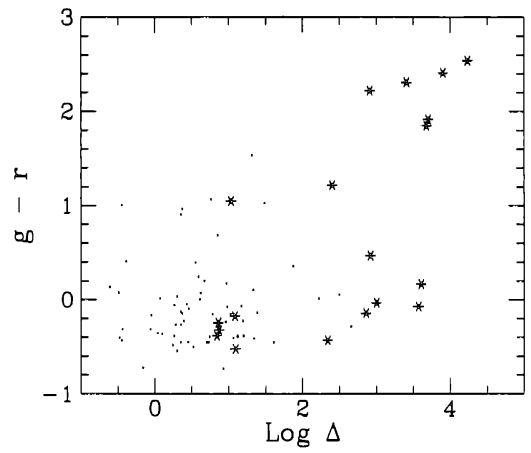

FIG. 3.-Color vs. local three-dimensional glob density contrast at $z=1$ for objects brighter than $r=25$. Objects in the cluster field of Fig. 1 are plotted as asterisks, and objects in random, noncluster fields as dots. the line of sight but outside the virial portion of the cluster. All globs with $\Delta>100$ in the cluster field are true cluster members. This population displays a nearly bimodal color distribution-blue members are objects on their first infall into the cluster (except for a merger product between an older cluster member and a freshly accreted, smaller glob at $g-r=0.4$ ), while red members have resided in the hot, intracluster environment for $\gtrsim 1$ Gyr. Examination of all globs in the simulation reveals that the reddest colors become bluer as $\Delta$ decreases. The maximum value of $g-r \simeq 2.3$ at $\Delta=300$ falls to $g-r \simeq 1.0$ at $\Delta=1$.

The different star-formation histories for objects in the cluster and random fields can be illustrated by plotting histograms of the ages of star particles within them. The age distributions, which reflect the cumulative star-formation history of globs in each population, are shown in Figure 4. The data have been normalized to the total number of star particles in the cluster field (7924 at $z=2$ and 12,859 at $z=1$ ). Gas accretion rates for individual globs at $z=1$ average $10 M_{\odot} \mathrm{yr}^{-1}$ in the field and are lower for cluster globs (Fig. 18 of ESD). The bulk of star formation occurs earlier in the cluster environment, the difference in median ages between the cluster and noncluster populations being $\sim 0.4 \mathrm{Gyr}$ at $z=2$ and $\sim 1 \mathrm{Gyr}$ at $z=1$. For the adopted cosmology, this translates into a peak of star formation around $2 \lesssim z \lesssim 4$ in the cluster environment, while star formation in random fields turns on around $z \approx 3$ and has declined more weakly by $z=1$. These differences are expected in cosmological models employing "bottoms-up" Gaussian random initial conditions, such as the CDM model adopted here. The underlying mechanism, sometimes called natural biasing (White et al. 1987), is one of accelerating the evolution of galactic scale perturbations in cluster environ-
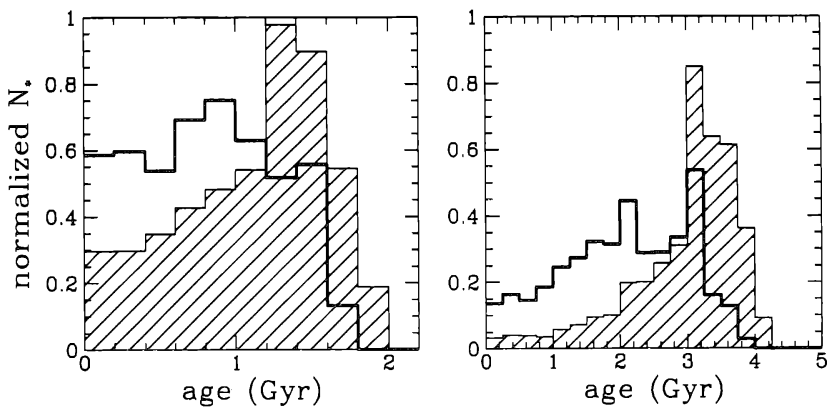

FIG. 4.-Histograms of the stellar population ages for objects within (shaded) and outside (unshaded) the cluster fields of Fig. 1 at (left) $z=2$ and (right) $z=1$ 
ments relative to their counterparts in the field (Bardeen et al. 1986; Cole \& Kaiser 1989).

\section{SUMMARY AND DISCUSSION}

We have investigated the expected optical appearance of a modest cluster of galaxies in a biased CDM universe by coupling a population synthesis model of stellar evolution with a combined $\mathrm{N}$-body/three-dimensional gas dynamic simulation of galaxy/cluster dynamics. Employing a simple star-formation criterion based on density and temperature thresholds intended to target highly dissipated baryons, we find that the cluster at $z=1$ contains bright red members whose accretion and star formation has been shut off at $z \simeq 2$ by interaction with the forming intracluster medium. No such red objects exist outside the cluster. The observed colors of some earlytype galaxies in high-redshift clusters may indicate that they have evolved passively since $z \simeq 2$ (Dressler \& Gunn 1990; Aragón-Salamanca et al. 1993). If, as our models suggest, the mechanism responsible for truncating the star-formation rate is interaction with a hot, intracluster medium, then observations of red galaxies in distant clusters imply the existence of hot intracluster gas in cluster cores at redshifts $z \simeq 2$. Direct detection of $X$-ray emission from such distant clusters may be possible in very deep exposures, since emission has been detected in $\sim 10 \mathrm{ks}$ ROSAT PSPC observations of clusters approaching $z \sim 1$ (Castander et al. 1994).

At higher redshift, the "red sequence" is less obvious because some amount of accretion and star formation has occurred recently for all objects. At $z=2$, the simulated objects appear very blue, and the difference in median colors (to a limiting $r=25 \mathrm{mag}$ ) between cluster and field is small, $\delta(g-r) \approx 0.1 \mathrm{mag}$.

The appearance of the simulated cluster at $z=2$ bears some resemblances to the collection of faint blue objects identified around a QSO at $z=2.055$ in a deep $H S T$ pointing toward the $z=0.4$ cluster CL $0930+4713$ by Dressler et al. (1993). Although redshifts for the faint, blue objects are not known, Dressler et al. speculated that they might be physically clus- tered with the $z=2$ QSO because of the small optical sizes of the objects and the fact that the QSO vicinity contained a significant excess of these objects above the background. If real, this would constitute, to our knowledge, the first of a cluster of galaxies at $z=2$.

Both the blue colors $(-0.1 \lesssim g-r \lesssim 0.4)$ and linear cluster morphology (see Fig. 1) are common to the observations and simulation. However, the overall richness and optical sizes of the galaxies are not well matched. Dressler et al. found an excess of roughly 8 galaxies brighter than $r=24.75$ within a $10^{\prime \prime}$ radius circle centered on the QSO. The simulated cluster contains five objects brighter than $r=25$ within the same radius measured from the cluster center. Presumably, simulating a richer cluster would reconcile this difference. The cluster in this simulation has a potential well depth a $2 \mathrm{keV}$. Naively, doubling the mass would double the count of galaxies and imply a modest temperature increase of a factor $2^{2 / 3}$ to $3.2 \mathrm{keV}$.

The other difference is the small physical extent, around 1 $\mathrm{kpc}$ radius, of the optically bright regions of the galaxies seen in the HST image. Dressler et al. postulate that these objects may in reality be subgalactic precursors due to merge into more familiar, present-day galaxies. The spatial and mass resolution of the present experiment is insufficient to resolve objects so small. Preliminary studies indicate that the amount of merging in the glob population may overestimate that expected for real galaxies (Evrard 1994). Future simulations with enhanced resolution may result in a larger number of smaller objects. At present, our work lends partial cautious support to Dressler et al.'s interpretation of this collection of faint blue objects as a cluster of "nascent" galaxies at $z=2$.

A. E. E. would like to thank his collaborators, F. J. Summers and $M$. Davis, for allowing use of the simulation data. This research was supported in part by the NASA Theory Program and the NSF by an allocation of supercomputer time at the San Diego Supercomputer Center and also in part by the US Department of Energy through Lawrence Livermore National Laboratory under contract No. W-7405-Eng-48.

Aragón-Salamanca, A., Ellis, R. S., Couch, W. J., \& Carter, D. 1993, MNRAS, 262,764

Bardeen, J. M., Bond, J. R., Kaiser, N., \& Szalay, A. S. 1986, ApJ, 304, 15

Baron, E., \& White, S. D. M. 1987, ApJ, 322, 585

Bertschinger, E. 1987, ApJ, 323, L103

Bruzual A, G., \& Charlot, S. 1993, ApJ, 405, 538 (BC)

Butcher, H., \& Oemler, A., Jr. 1978, ApJ, 219, 18

Castander, F. J., Ellis, R. S., Frenk, C. S., Dressler, A., \& Gunn, J. E. 1994, ApJ,

in press
Cen, R., \& Ostriker, J. P. 1992, ApJ, 393, 22

Charlot, S., \& Silk, J. 1993, ApJ, submitted

Cole, S., \& Kaiser, N. 1989, MNRAS, 237, 1127

Dekel, A., \& Silk, J. 1986, ApJ, 303, 39

Dickinson, M. 1993, in preparation

REFERENCES

Dressler, A., \& Gunn, J. E. 1990, in The Evolution of the Universe of Galaxies, ed. R. G. Kron (San Francisco: ASP), 200 .1992, ApJS, 78, 1

Dressler, A., Oemler, A., Jr., Gunn, J. E., \& Butcher, H. 1993, ApJ, 404, L45 Efstathiou, G. 1992, MNRAS, 256, 43P

Evrard, A. E. 1994, in Modeling the Dynamics of the Universe, ed. J. Franco (Cambridge: Cambridge Univ. Press), in press

Evrard, A. E., Summers, F. J., \& Davis, M. 1994, ApJ, 422, 11 (ESD)

Hintzen, P., Romanishin, W., \& Valdes, F. 1991, ApJ, 366, 7

Kaiser, N. 1986, MNRAS, 222, 323

Katz, N. 1992, ApJ, 391, 502

Larson, R. B., Tinsley, B. M., \& Caldwell, C. N. 1980, ApJ, 237, 692

White, S. D. M., Frenk, C. S., Davis, M., \& Efstathiou, G. 1987, Nature, 330, 451 


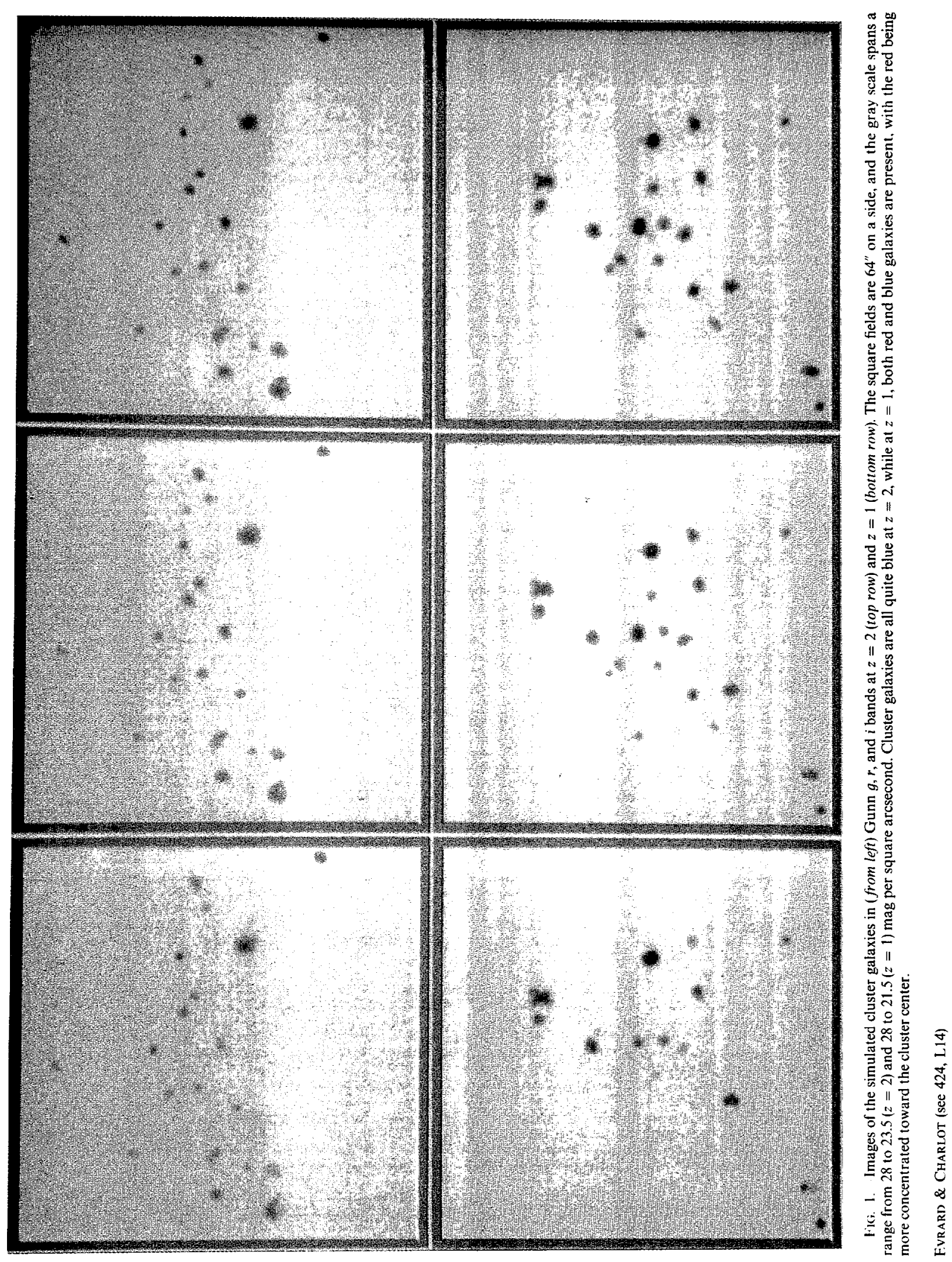

